\title{
FABRICATION AND TEST OF THE FIRST NORMAL-CONDUCTING CROSSBAR H-TYPE ACCELERATING CAVITY AT FERMILAB FOR HINS*
}

\author{
L. Ristori”, G. Apollinari, I. Gonin, T. Khabiboulline, G. Romanov, Fermilab, Batavia, IL 60510.
}

\begin{abstract}
The proposed High Intensity Neutrino Source (HINS) at Fermilab is based on an $8 \mathrm{GeV}$ linear proton accelerator that consists of a normal-conducting (warm) and a superconducting section.

The warm section is composed of an ion source, a radio frequency quadrupole, a medium energy beam transport (MEBT) and 16 warm Crossbar H-type (CH) cavities that accelerate the beam from $2.5 \mathrm{MeV}$ to $10 \mathrm{MeV}$ (from $\beta=0.0744$ to $\beta=0.1422$ ). These warm cavities are separated by superconducting solenoids enclosed in individual cryostats. Beyond $10 \mathrm{MeV}$, the design uses superconducting spoke resonators to accelerate the beam up to $8 \mathrm{GeV}$.

In this paper, we illustrate the completion of the first warm $\mathrm{CH}$ cavity $(\beta=0.0744)$ explaining in detail the mechanical engineering aspects related to the machining and brazing processes. The radio-frequency (RF) measurements and tuning performed at Fermilab on the resonator and comparisons with simulations are also discussed.
\end{abstract}

\section{INTRODUCTION}

The Fermilab HINS is an $8 \mathrm{GeV}$ superconducting $\mathrm{H}^{-}$ LINAC with the primary mission of enabling $2 \mathrm{MW}$ beam power from the $120 \mathrm{GeV}$ Fermilab Main Injector for a neutrino program. Fermilab's approach in this development is to align this effort with the laboratory's International Linear Collider (ILC) strategy.

Superconducting cavities operating at $1300 \mathrm{MHz}$ and originally developed for the electron-positron linear collider can be directly applied for acceleration of $\mathrm{H}^{-}$or proton beams above $1.2 \mathrm{GeV}$. Squeezed ILC-style cavities designed for $\beta=0.81$ can be used in the energy range from $\sim 400 \mathrm{MeV}$ to $1.2 \mathrm{GeV}$. The front end LINAC in the energy range from $10 \mathrm{MeV}$ to $400 \mathrm{MeV}$ is a section operating at the 4th sub-harmonic of the ILC frequency and is based on $325 \mathrm{MHz}$ superconducting spoke resonators [1].

One of the new paradigms introduced into the front end design is the adoption of short, high field superconducting solenoids as primary lattice focusing elements, and a low energy transition at $10 \mathrm{MeV}$ from room temperature (RT) to superconducting $\mathrm{RF}$ acceleration. To provide adiabatic variation of the wave numbers, accelerating gradient has to change from $\sim 0.75$ $\mathrm{MV} / \mathrm{m}$ - at the radio frequency quadrupole (RFQ) end -

\footnotetext{
*This work was supported by the U.S. Department of Energy under contract number DE-AC02-76CH03000.

"leoristo@fnal.gov.
}

to $\sim 2 \mathrm{MV} / \mathrm{m}-$ at the beginning of superconducting section. This can be achieved by using short RT accelerating cavities incorporated into a solenoidal focusing lattice up to $\sim 10 \mathrm{MeV}$. This reduces the number of different types of superconducting cavities and provides adiabatic beam matching. By focusing the beam using superconducting solenoids we obtain a more compact lattice and a shorter focusing period.

Our studies show that the most appropriate RT accelerating structure in the energy range $2.5-15 \mathrm{MeV}$ is a $\mathrm{CH}$ cavity [2,3] operating at $325 \mathrm{MHz}$.

We have successfully fabricated, fully dressed and tested at low power the first resonator which is now ready for high power tests. We are currently fabricating $\mathrm{CH} 2$ $(\beta=0.0771), \mathrm{CH} 3 \quad(\beta=0.0804)$ and $\mathrm{CH} 4 \quad(\beta=0.0842)$ resonators in parallel.

\section{RF DESIGN AND OPTIMIZATION OF THE RT CH RESONATORS}

In general, H-type cavities are highly effective in the low energy range. However, the current HINS LINAC design is based on the scheme with RF power fan-out from one klystron to multiple cavities, which puts additional limitations on power consumption by the RT and the other accelerating sections. Besides that, the focusing lattice makes our $\mathrm{CH}$ cavities very short, with only 4 or 5 accelerating gaps, and for short H-type cavities the shunt impedance is significantly reduced. Therefore it was especially important for our RT $\mathrm{CH}$ cavities to maximize the shunt impedance. The RT accelerating section between the MEBT and the superconducting part of the accelerator comprises 16 cavities (four 4-gap cavities and twelve 5-gap cavities). The beam energy from $2.5 \mathrm{MeV}$ is accelerated up to 10 $\mathrm{MeV}$. Each cavity consists of 3-4 identical cells with geometrical beta corresponding to the relevant beam velocity at the cavity mid plane. Moreover, in order to simplify production, all cavity drift tubes are identical (except for their length) and cross-bars of spokes have the same basic shape. A more detailed RF design and optimization flow is given in [3]. The only change since the RF design was complete is that the first cavity operates now as an additional buncher. This didn't modify either the RF or the mechanical design.

\section{FABRICATION OF RESONATOR CH-1}

The machining process for the spokes of the first resonator was fine-tuned working on aluminum samples before using oxygen-free electrolytic copper (OFE-Cu). 
The overall machining and finishing of the spokes was successful and demonstrated the feasibility of copper spokes machined from solid with tolerances down to \pm 0.001 inch and average surface roughness under $63 \mu$ in (Fig. 1).

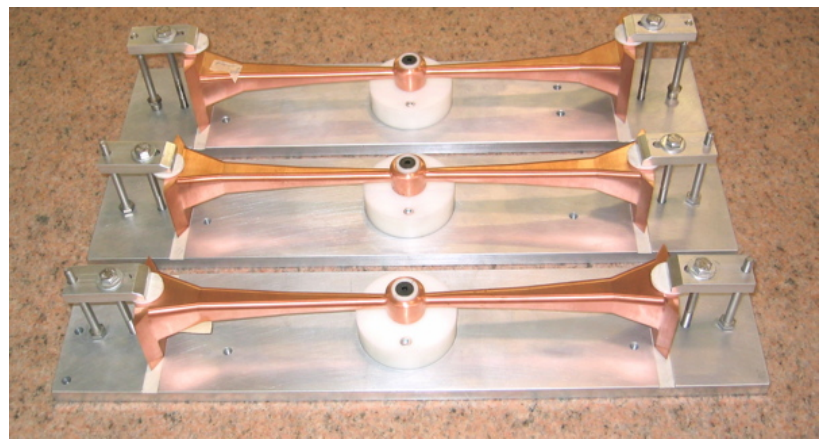

Fig. 1: The three OFE-Cu spokes finished and ready for assembly inside the resonator.

To install the 3 spokes inside the cylinder (also made of solid OFE-Cu) vacuum furnace brazing was chosen using a 50-50 $\mathrm{Au}-\mathrm{Cu}$ alloy, all the stainless steel ports on the cylinder were previously brazed using a 35-65 $\mathrm{Au}-\mathrm{Cu}$ alloy (Fig. 2). Shoulder screws were used to secure the spokes to the cylinder in order to obtain a better contact around the bases and also provide correct positioning. Threaded inserts were installed on the spoke bases to provide a more reliable joint.

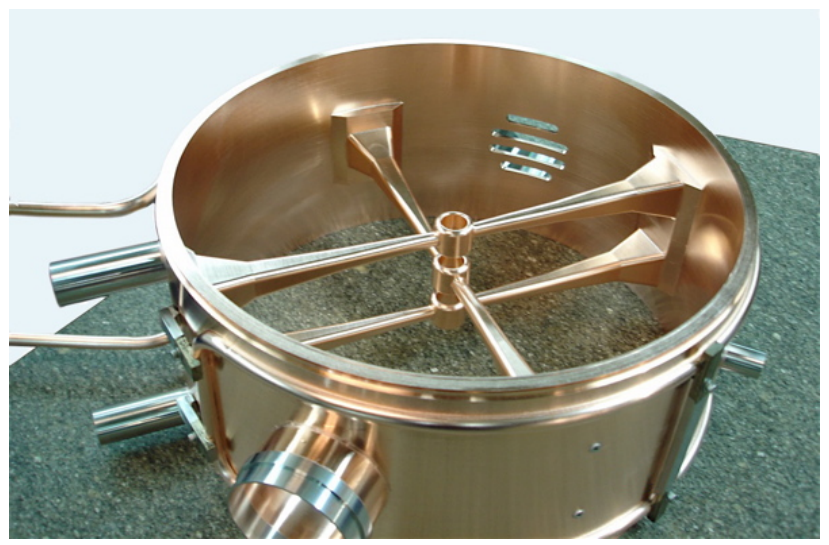

Fig. 2: The resonator after brazing of the spokes and ready for welding of the flanges. Two shoulder screws are visible on the bottom right.

The connection between the copper wall and the external steel wall (separated by a $2 \mathrm{~mm}$ air gap to insulate the copper walls from atmospheric pressure) was also brazed using a 35-65 Au-Cu alloy. The two doublelayer end walls were later welded on the main body with the adoption of a $0.2 \mathrm{~mm}$ protruding lip to obtain a good RF contact after weld shrinkage.

Two plunger-type tuners with copper fingers of $20 \mathrm{~mm}$ in diameter and a stroke of $50 \mathrm{~mm}$ will be used to control the frequency of the resonator in a feedback loop system (Fig. 4).

\section{FABRICATION OF INPUT COUPLERS}

Two prototypes for the input coupler [3] were successfully fabricated and tested. The custom made ceramic window was brazed to two copper sleeves that later were brazed to the inner and outer conductors of the coupler. Shoulder screws were used to secure the coupler tip during brazing (Fig. 5).

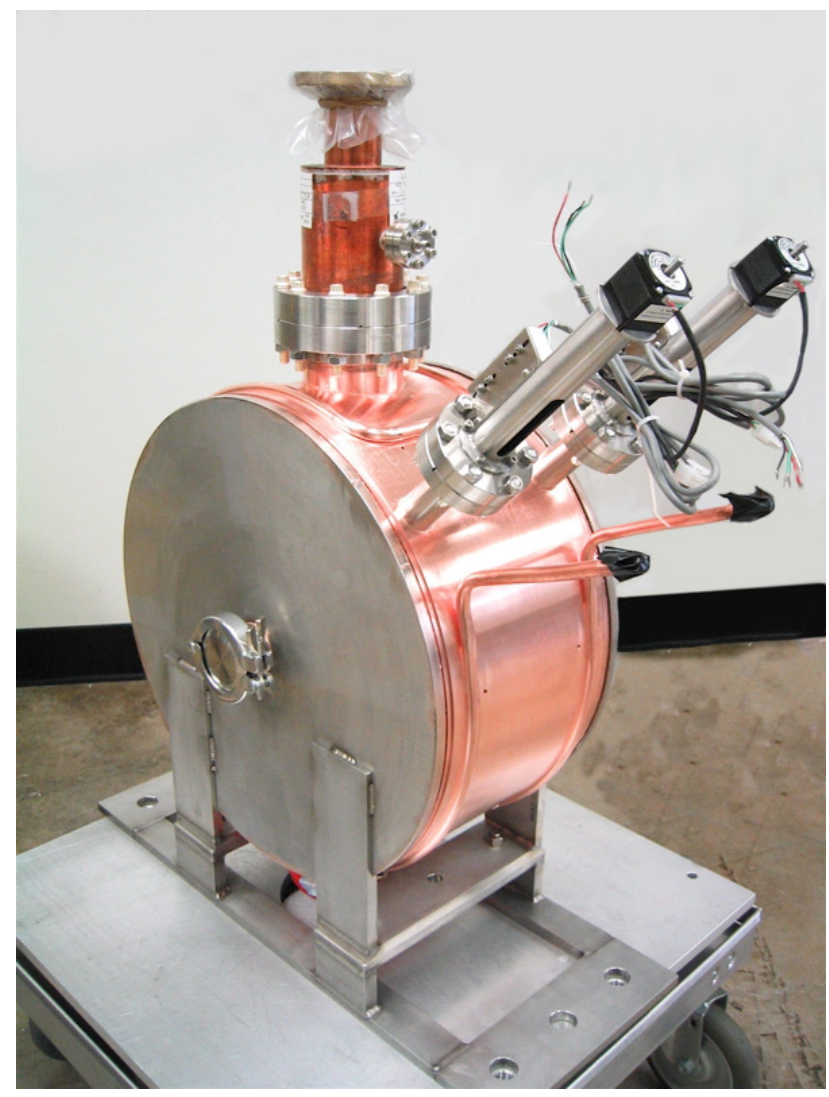

Fig. 3: The fully dressed prototype, the universal stand is visible at the bottom bolted to the resonator's support brackets. The same stand is used for all the resonators.

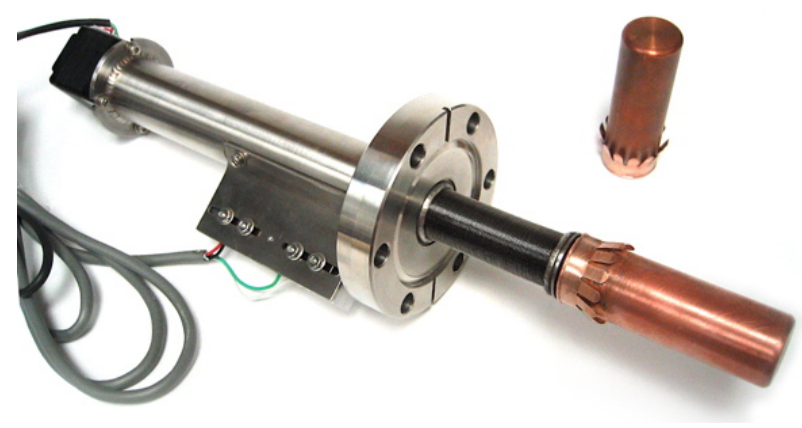

Fig. 4: One of two prototype tuners used on the resonator, $\mathrm{Be}-\mathrm{Cu}$ contacts are welded to the removable copper fingers, hard stops and limit switches define the stroke, a linear actuator with captive shaft is used to operate the tuner. 


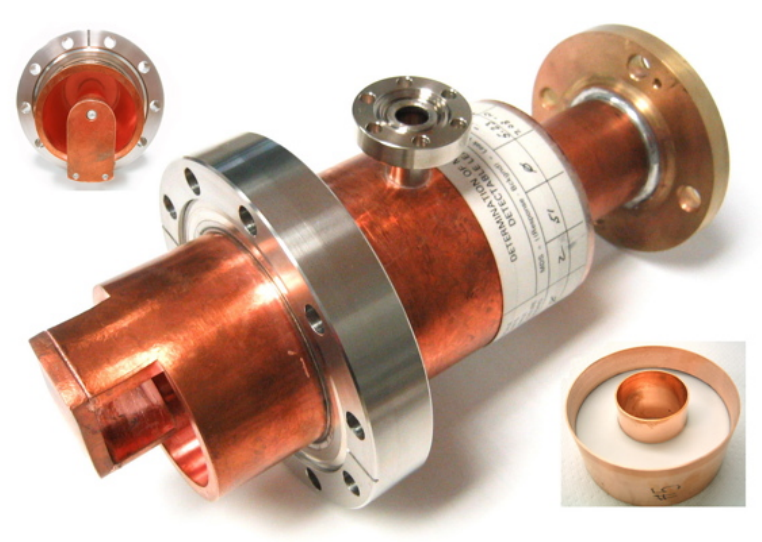

Fig. 5: One of the two finished input couplers.

\section{MEASUREMENTS}

The first measurements on the prototype for $\mathrm{CH} 1$ were performed clamping the end walls on the main body before the final welding. At this stage it was seen that brazing of the spokes had been successful.

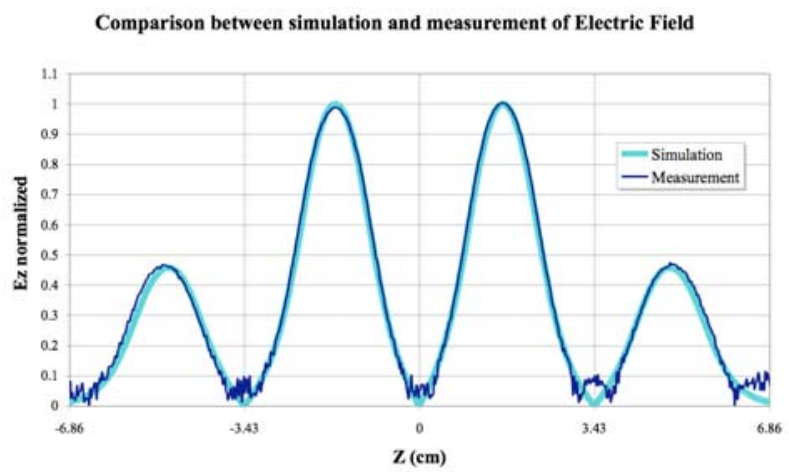

Fig. 6: Comparison between the simulated and measured accelerating electric field along the beam axis before final welding of the resonator. The period of the resonator is $3.43 \mathrm{~cm}$.

After final welding, the measured operating frequency decreased by $2.2 \mathrm{MHz}$. Subsequent mechanical (Fig. 7) and RF measurements indicated that this was due to the displacement of one spoke. Since a shock test showed the low sensitivity of the resonator's operating mode frequency to transportation, the cause of the damage was attributed to mishandling during the fixturing operations for final welding.

The tuning of the damaged spoke was relatively easy due to the ductility of the annealed material. The target frequency and a symmetrical accelerating field were achieved in a few iterations using a simple tool.

The resonator was later put under vacuum to measure its behavior under the differential pressure of 1 atmosphere. With a measured shift of $+43 \mathrm{kHz}$, the contribution due to mechanical deformations is calculated to be $-58 \mathrm{kHz}$, in accordance with former structural simulations $(+101 \mathrm{kHz}$ is taken into account as the change due to the difference of air and vacuum properties).

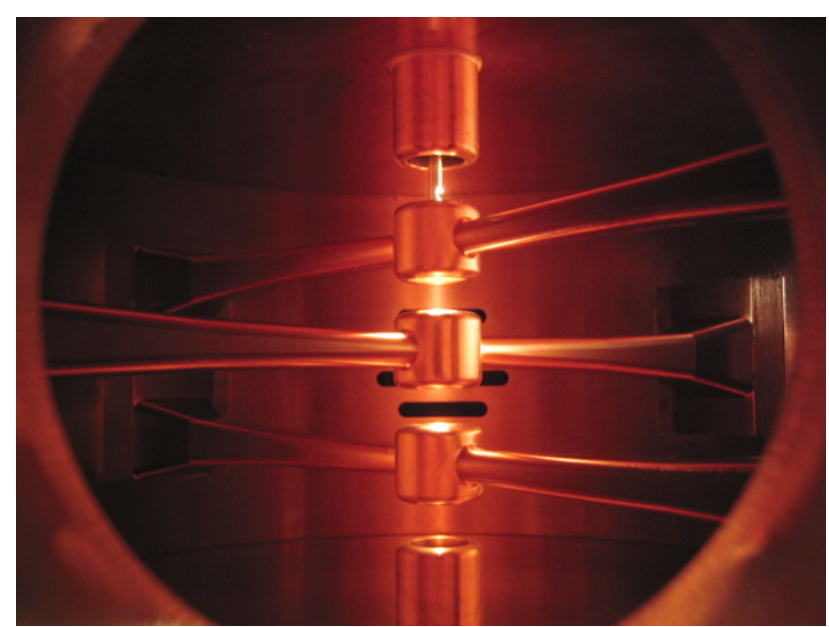

Fig. 7: Inside view of the resonator from the RF port, a borescope with its light source used for mechanical measurements is visible at the top. The vacuum grill is visible behind the drift tubes.

The two plunger-type tuners were installed and their tuning capabilities were assessed to a tuning range of \pm $0.6 \mathrm{MHz}$ which follows the predicted values. The installation of the input coupler generated a frequency shift of $+25 \mathrm{kHz}$ in accordance with the predicted value.

The rotatable flange on the coupler allows the adjustment of the coupling, the maximum coupling is achieved with the loop perpendicular to the beam axis, the maximum recorded value was $\mathrm{Q}_{\text {ext }}=8800$.

A loop type pick-up probe was installed on the cylinder at 90 degrees with the coupler and adjusted to have a $6 \mathrm{~W}$ signal power and $\mathrm{Q}_{\mathrm{ext}}=10^{-7}$.

\section{CONCLUSIONS}

The RF and mechanical designs have proven to be successful. The completion of the first cavity, two input couplers and two plunger tuners, demonstrated the overall feasibility of the fabrication process. Resonator $\mathrm{CH} 1$ is fully dressed for the first high power tests. Resonators $\mathrm{CH} 2, \mathrm{CH} 3$ and $\mathrm{CH} 4$ are being procured and the design for the remaining resonators $\mathrm{CH} 5-\mathrm{CH} 16$ is in process.

\section{ACKNOWLEDGEMENTS}

Special thanks to: L. Alsip, T. Beale, L. Elementi, R. Evans, O. Lira, L. Simmons and J. Williams.

\section{REFERENCES}

[1] P.N. Ostroumov et al, "Front End Design of a MultiGeV H-Minus Linac", PAC-2005, Knoxville, Tennessee, May 2005.

[2] Z. Li, R. Tiede, U. Ratzinger et al., "Design of the R.T. CH-Cavity and Perspectives for a New GSI Proton Linac", LINAC-2004, Lubeck, August 2004.

[3] L. Ristori, G. Romanov, T. Khabiboulline et al., "Design of normal conducting $325 \mathrm{MHz}$ crossbar htype resonators at Fermilab", LINAC-2006, Knoxville, Tennessee, August 2006. 